\title{
Silencing the PIK3CA Gene Enhances the Sensitivity of Childhood Leukemia Cells to Chemotherapy Drugs by Suppressing the Phosphorylation of Akt
}

\author{
Xiuling Liang ${ }^{1}$ Xianfang Xin ${ }^{1}$, Dongmei $\mathrm{Qi}^{1}$, Chengyan $\mathrm{Fu}^{1}$, and Mingde Ding ${ }^{2}$ \\ Departments of ${ }^{1}$ Pediatric Internal Medicine and ${ }^{2}$ Gynecology, Affiliated Hospital of Taishan Medical University, Tai'an, China.
}

\begin{abstract}
Purpose: This study aimed to investigate the effects of PIK3CA on the sensitivity of acute B lymphocytic leukemia cells (Nalm-6 cells) to chemotherapy drugs.

Materials and Methods: Children's normal B lymphocytes and Nalm-6 cells were cultured. Nalm-6 cells were transfected with PIK3CA siRNA (siPIK3CA group) or its negative control (PIK3CA-Control group). Normal Nalm-6 cells were named Mock group. Nalm- 6 cells transfected by PIK3CA siRNA were treated with Akt inhibitor (siPIK3CA+Akti-1/2 group). mRNA and protein expression was detected by qRT-PCR and Western blot. Proliferation and sensitivity to chemotherapeutic drugs was detected by MTT assay. Cell cycle and apoptosis was explored by low cytometry. Transwell assay was performed to test invasion.

Results: PIK3CA mRNA ( $p=0.008)$ and protein $(p=0.006)$ expression was higher in Nalm- 6 cells than that in normal B lymphocytes. Compared with the Mock group and PIK3CA-Control group, Nalm-6 cells of the siPIK3CA group had lower OD495 values (all $p<0.05)$ and invasion cell numbers ( $p=0.03$ and $p=0.025)$, as well as a higher proportion of G0/G1 phase cells ( $p=0.020$ and $p=$ 0.022 ), percentage of apoptosis ( $p=0.016$ and $p=0.022)$, and inhibition rate (all $p<0.05)$. pAkt expression in the siPIK3CA group $(p=$ 0.026 and $p=0.031$ ) and siPIK3CA+Akti-1/2 group ( $p=0.019$ and $p=0.023$ ) was lower than that in the Mock group.

Conclusion: PIK3CA silencing inhibited Nalm-6 cell proliferation and invasion, and promoted their apoptosis and sensitivity to chemotherapeutic drugs, potentially through regulation of the PI3K/AKT signaling pathway.
\end{abstract}

Key Words: PIK3CA, childhood leukemia cells, proliferation, apoptosis, invasion

\section{INTRODUCTION}

Leukemia is a type of hematopoietic stem cell malignant clonal disease that often occurs in childhood and is accompanied by defects, such as uncontrolled proliferation and blocked apoptosis of leukemia cells. ${ }^{1,2}$ Leukemia has been divided into acute leukemia and chronic leukemia, with a large proportion of

Received: August 22, 2018 Revised: November 30, 2018

Accepted: December 4, 2018

Corresponding author: Mingde Ding, MBBS, Department of Gynecology, Affiliated Hospital of Taishan Medical University, No. 706, Taishan Street, Tai'an, Shandong 271000 , China.

Tel: 86-0538-623-7469, Fax: 86-0538-842-0042, E-mail: dingmingde24@163.com

-The authors have no potential conflicts of interest to disclose.

(C) Copyright: Yonsei University College of Medicine 2019

This is an Open Access article distributed under the terms of the Creative Commons Attribution Non-Commercial License (https://creativecommons.org/licenses/ by-nc/4.0) which permits unrestricted non-commercial use, distribution, and reproduction in any medium, provided the original work is properly cited. children diagnosed with acute lymphoblastic leukemia., ${ }^{3,4}$ Epidemiological studies in recent years have shown an increased incidence of childhood leukemia, which might be related to aggravated environmental and air pollution. ${ }^{5}$ Meanwhile, leukemia treatment has remained an urgent problem for medical researchers, because of its serious life threating effects to patients, especially children.

At present, the treatment of leukemia is based on comprehensive chemotherapy-based treatment. Chemotherapy drugs can affect DNA transcription and inhibit the synthesis of nucleic acids and proteins, depending on their effective molecular targets, thereby inducing apoptosis of leukemia cells. ${ }^{6,7} \mathrm{How}$ ever, like many other malignant cells, leukemia cells can escape apoptosis through many mechanisms, such as overexpressing apoptotic inhibitory proteins, which are causes of primary resistance and secondary resistance to chemotherapeutic drugs and ultimately lead to chemotherapy failure., ${ }^{8,9}$ Therefore, improving the sensitivity of leukemia cells to chemotherapeu- 
tic drugs is of great significance for improving the prognosis of leukemia patients.

With the rapid development of life science research, some important genes have been found to possess the ability to increase the chemosensitivity of leukemia cells. Wang, et al. $.^{10} \mathrm{ob}-$ served from their research that reduced nucleophosmin/B23 expression enhances leukemia cells sensitivity to Adriamycin by regulating the Akt/mTOR signaling pathway. Xu, et al. ${ }^{11} \mathrm{in}$ dicated that up-regulation of SIRT2 could impair multidrug sensitivity among acute myeloid leukemia cells, while the suppression of ERK1/2 could reverse drug resistance in these cells upon SIRT2 up-regulation. Jiang, et al. ${ }^{12}$ described enhanced sensitivity to cytarabine and vincristine by the lymphocytic leukemia cell lines Reh and RS4:11 upon up-regulation of miR652-3p. Liang, et al. ${ }^{13}$ demonstrated that acute myeloid leukemia cell drug sensitivity was attenuated by GLIl inhibition, along with a possible potential mechanism of promoting AKT phosphorylation.

PIK3CA mutation has been found to result in progression of a variety of tumors and thus has been considered an oncogene. Intervention of the cell cycle is one of the main means by which PIK3CA promotes tumor progression. Gu, et al. ${ }^{14}$ researched aspirin effects on three different PIK3CA-mutant colon cancer cell lines (SW948, HCT15, and HCT1160). Their results indicated that these three cell lines all exhibit significant sensitivity to aspirin: most cells were arrested at G0/G1 phase after exposure to aspirin for $48 \mathrm{~h}$ and $72 \mathrm{~h}$. Zheng, et al. ${ }^{15}$ revealed that, after PIK3CA is silenced, esophageal squamous cell carcinoma cells show impaired proliferation, migration, and invasion abilities, in addition to enhanced apoptosis ability. Meanwhile, PIK3CA silencing blocked most esophageal squamous cell carcinoma cells at S phase. Nevertheless, PIK3CA expression in leukemia and its impact on leukemia chemotherapy have not been studied. In this research, the effects of PIK3CA knockdown on the proliferation, cell cycle, invasion, and apoptosis of leukemia cells were studied, along with its effects on drug sensitivity and potentially related mechanisms.

\section{MATERIALS AND METHODS}

\section{Cell culture}

Children's normal B lymphocytes and the acute B lymphocytic leukemia cell line Nalm-6 were purchased from Wuhan Cell Bank (Wuhan, China). Both cells lines were cultured in RPMI1640 complete medium containing $10 \%$ fetal bovine serum (FBS), $100 \mathrm{U} / \mathrm{mL}$ of penicillin, and $100 \mathrm{U} / \mathrm{mL}$ of streptomycin at $37^{\circ} \mathrm{C}, 5 \% \mathrm{CO}_{2}$. The medium was replaced every two days, and cells were passaged after being digested with $0.25 \%$ trypsin at about $80 \%$ confluence. This study obtained ethical committee approval of Affiliated Hospital of Taishan Medical University.

\section{Cell transfection}

PIK3CA siRNA and its negative control sequence were synthesized by Shanghai Shenggong Bioengineering Co., Ltd. (Shanghai, China). PIK3CA in Nalm-6 cells was silenced by RNA interference technology using Lipofectamine 2000 kits (Thermo Fisher Scientific, Waltham, MA, USA). Briefly, Nalm-6 cells were seeded in 6-well plates containing serum-free RPMI-1640 medium and transfected by PIK3CA siRNA (set as siPIK3CA group) or its negative control (served as PIK3CAControl group). After $6 \mathrm{~h}$ incubation at $37^{\circ} \mathrm{C}, 5 \% \mathrm{CO}_{2}$, residual liquid in each well was discarded and replaced by RPMI- 1640 complete medium (containing 10\% FBS) for continued incubation. Nalm- 6 cells without any treatment were used as a Mock group. In addition, Nalm- 6 cells transfected by PIK3CA siRNA were subjected to culture with RPMI-1640 complete medium containing both $10 \%$ FBS and $5 \mu \mathrm{mol} / \mathrm{L}$ of Akti-1/2 (Akt inhibitor, Sigma, San Francisco, CA, USA) as a siPIK3CA+ Akti-1/2 group. All cells were cultured under $37^{\circ} \mathrm{C}, 5 \% \mathrm{CO}_{2}$.

\section{qRT-PCR detection}

RNA purity was detected after RNA was extracted from cells by TRIzol reagent (Thermo Fisher Scientific). Reverse transcription using a PCR instrument was conducted to synthesize cDNA templates. Using an ABI7500 quantitative PCR instrument (Applied Biosystems, Foster City, CA, USA), real-time fluorescence quantitative PCR was carried out under the following conditions: pre-denaturation at $95^{\circ} \mathrm{C}$ for $10 \mathrm{~min}, 38$ cycles of denaturation at $95^{\circ} \mathrm{C}$ for $30 \mathrm{~s}$, annealing at $60^{\circ} \mathrm{C}$ for $20 \mathrm{~s}$, and extension at $72^{\circ} \mathrm{C}$ for $34 \mathrm{~s}$. The primers used were as follows: PIK3CA, forward, 5'-CACTGAGCAGGAGAAAGAT-3', reverse, 5'-CAGTCCAGAAGTTCCATAGC-3'; GAPDH, forward, 5'-ATA AATTGAGCCCGCAGCC-3', reverse, 5'-ACCAAATCCGTTGA CTCCGA-3'. GAPDH served as an internal reference. Results were analyzed by the $2^{-\Delta \Delta \mathrm{Ct}}$ method.

\section{Western blot}

Proteins were separated through sodium dodecyl sulfate polyacrylamide gel electrophoresis (SDS-PAGE) after being extracted from cells, followed by being transferred onto a PVDF membrane for $5 \%$ skim milk-TBST closure. Membranes were then rinsed three times with TBST. Primary antibodies (rabbit anti-human PIK3CA, AKT, pAKT, 1:1000, Cell Signaling, Boston, MA, USA) were used to incubate membranes for $12 \mathrm{~h}$ at $4^{\circ} \mathrm{C}$, followed by $1 \mathrm{~h}$ incubation with horseradish peroxidaselabeled goat anti-rabbit IgG (1:5000, Beijing ComWin Biotech Co., Ltd., Beijing, China). After rinsing the membranes another three times with TBST, an ECL chemiluminescence system (Bio-Rad, Hercules, CA, USA) was used to visualize immunoreactive complexes. GAPDH was set as an internal reference.

\section{MTT assay}

Under $37^{\circ} \mathrm{C}, 5 \% \mathrm{CO}_{2}$, 96-well plates inoculated with cells of each group were incubated for 1-4 days. Cells proliferation 
was measured on days $1,2,3$, and 4 . In short, $20 \mu \mathrm{L}$ of MTT solution ( $5 \mathrm{mg} / \mathrm{mL}$ ) was added to each well, and $4 \mathrm{~h}$ later, the residual liquid was discarded and replaced with $150 \mu \mathrm{L}$ of DMSO. After the formazan was fully dissolved, these 96-well plates were placed on a microplate reader to determine absorbance at $495 \mathrm{~nm}$ (OD495 value).

\section{Cell cycle detection by flow cytometry}

After being cultured for 4 days, cells were fixed with precooled $70 \%$ ethanol. PBS was used to wash these cells twice. Cells at the bottom of the centrifuge tube were collected after being centrifuged and were resuspended with Muse ${ }^{\mathrm{TM}}$ Cell Cycle Reagent for $30 \mathrm{~min}$ incubation at room temperature in the dark. A Muse ${ }^{\mathrm{TM}}$ Cell Analyzer (Merck Millipore, Billerica, MA, USA) was used to detect cell cycle distribution.

\section{Apoptosis detection by AnnexinV-PI staining}

At $48 \mathrm{~h}$, cells were collected and centrifuged at $2000 \mathrm{rpm}$ for 5 min after being rinsed twice with PBS. A total of $500 \mu \mathrm{L}$ of binding buffer and $5 \mu \mathrm{L}$ of propidium iodide was added to suspend cells, followed by $10 \mathrm{~min}$ incubation at room temperature in the dark. Apoptosis was detected by flow cytometry at an excitation wavelength of $488 \mathrm{~nm}$ and an emission wavelength of $530 \mathrm{~nm}$.

Invasion ability detection by Transwell assay

Cells suspended in serum-free RPMI-1640 medium were in- oculated into Transwell chambers (pre-coated with a layer of Matrigel) at a density of $2 \times 10^{5}$ cells $/ \mathrm{mL}$. Each Transwell chamber was inoculated with $200 \mu \mathrm{L}$ of cell suspension. A total of $400 \mu \mathrm{L}$ of RPMI-1640 medium containing $20 \%$ FBS was added into the lower chamber. Cells were cultured at $37^{\circ} \mathrm{C}, 5 \% \mathrm{CO}_{2}$ for $48 \mathrm{~h}$. Cells on the upper surface of the membrane were wiped off with a cotton swab, while those passing through the membrane were fixed with $4 \%$ paraformaldehyde for $30 \mathrm{~min}$ and stained with crystal violet for $20 \mathrm{~min}$. Five randomly different fields of view under the microscope were selected to calculate the number of invading cells.

\section{Sensitivity of cells to chemotherapy drugs}

Changes in cell sensitivity to chemotherapeutic drugs were detected using the MTT assay. In short, Nalm- 6 cells were cultured in RPMI-1640 medium (10\% FBS) containing $0.5 \mu \mathrm{g} / \mathrm{mL}$ of vincristine (VCR) and $0.5 \mu \mathrm{g} / \mathrm{mL}$ of daunorubicin (DNR), respectively. In this study, the Control group was also set with only adding RPMI-1640 medium (10\% FBS) containing the same concentration of different drugs in each well. On the first, second, third, and fourth day after being cultured at $37^{\circ} \mathrm{C}, 5 \%$ $\mathrm{CO}_{2}$, the absorbance value of each well at a wavelength of 495 nm (OD495 value) was measured using the MTT method as described above. The inhibition rate was calculated according to the following formula: Inhibition rate $=[1-$ (OD495 value of experimental group/OD495 value of Control group)]×100\%.

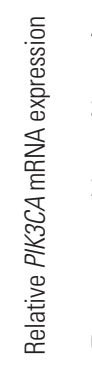

A

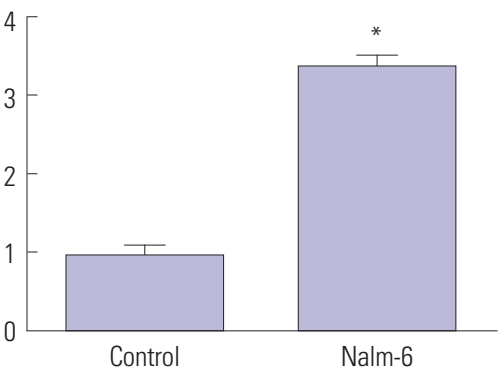

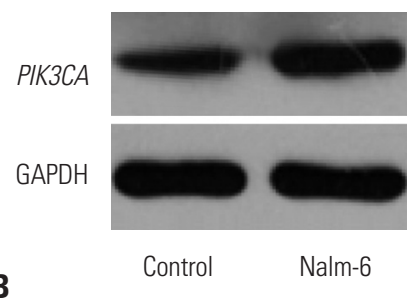

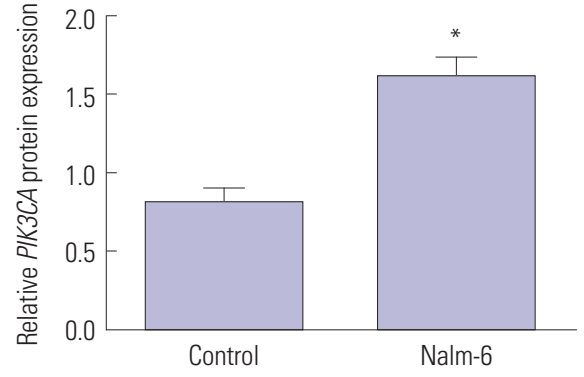

Fig. 1. PIK3CA up-regulated in Nalm-6 cells. (A) PIK3CA mRNA was up-regulated in Nalm-6 cells. (B) PIK3CA protein was increased in Nalm-6 cells. ${ }^{*} p<0.01$.

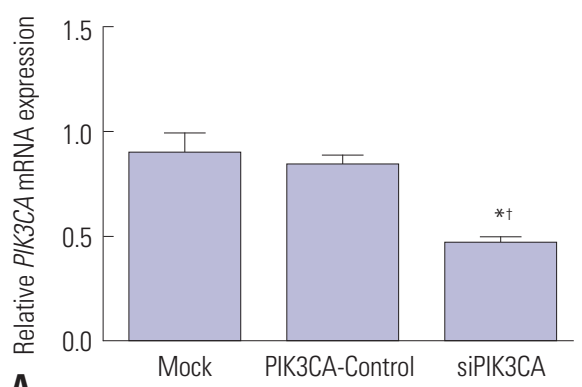

A

Fig. 2. PIK3CA silenced in Nalm-6 cells by siPIK3CA transfection. (A) PIK3CA siRNA transfection inhibited PIK3CA mRNA expression in Nalm-6 cells. (B) PIK3CA siRNA transfection suppressed PIK3CA protein expression in Nalm- 6 cells. ${ }^{*} p<0.01$ when compared with Mock group, ${ }^{\dagger} p<0.01$ when compared with PIK3CA-Control group. 


\section{Statistical analysis}

Data statistical analysis was performed using SPSS 13.0 software (SPSS Inc., Chicago, IL, USA) with GraphPad Prism 5.0 (GraphPad Software, San Diego, CA, USA) software as the drawing software. Data were analyzed as means \pm standard deviations, and Student's t-test was selected to analyze differences between two groups. $p<0.05$ was considered statistically significant.

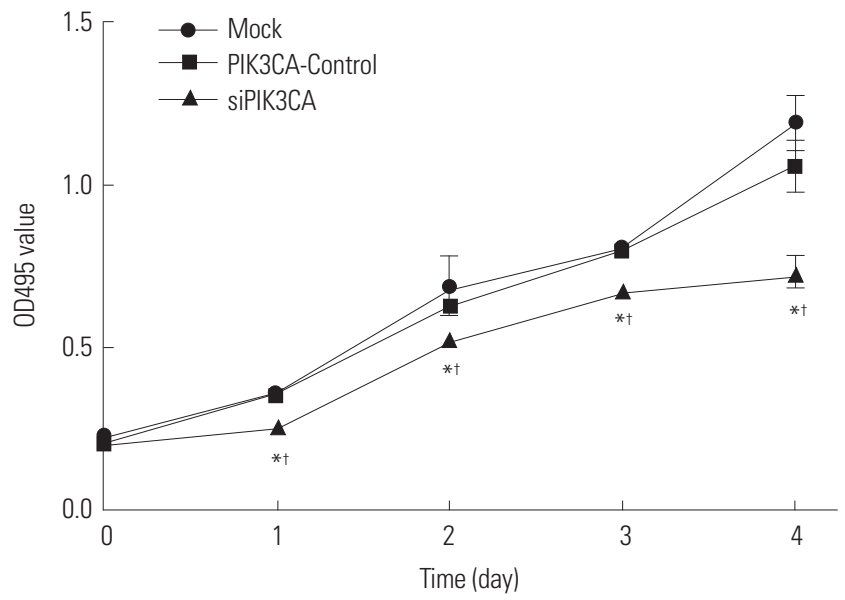

Fig. 3. PIK3CA silencing inhibits Nalm- 6 cell proliferation. * $p<0.05$ when compared with Mock group at the same time, ${ }^{\dagger} p<0.05$ when compared with PIK3CA-Control group at the same time.

\section{RESULTS}

\section{PIK3CA up-regulated in Nalm-6 cells}

PIK3CA expression in children's normal B lymphocytes (set as a Control group) and Nalm-6 cells (set as Nalm-6 group) was measured by qRT-PCR and Western blot. In result, PIK3CA mRNA ( $p=0.008)$ and protein $(p=0.006)$ relative expression was both significantly increased in the Nalm-6 group when compared to the Control group (Fig. 1), illustrating upregulation of PIK3CA in Nalm- 6 cells.

\section{PIK3CA silenced in Nalm-6 cells by siPIK3CA transfection}

After being transfected, the relative PIK3CA mRNA ( $p=0.003)$ and protein $(p=0.0026)$ expression in Nalm- 6 cells of the siPIK3CA group was markedly reduced when compared to the Mock group. Meanwhile, when compared with the PIK3CA-Control group, Nalm- 6 cells of the siPIK3CA group also showed much lower relative PIK3CA mRNA ( $p=0.004)$ and protein $(p=0.0029)$ expression. No significant difference was found in relative PIK3CA mRNA and protein expression between the PIK3CA-Control group and Mock group (Fig. 2). PIK3CA siRNA transfection could effectively silence PIK3CA expression in Nalm-6 cells.
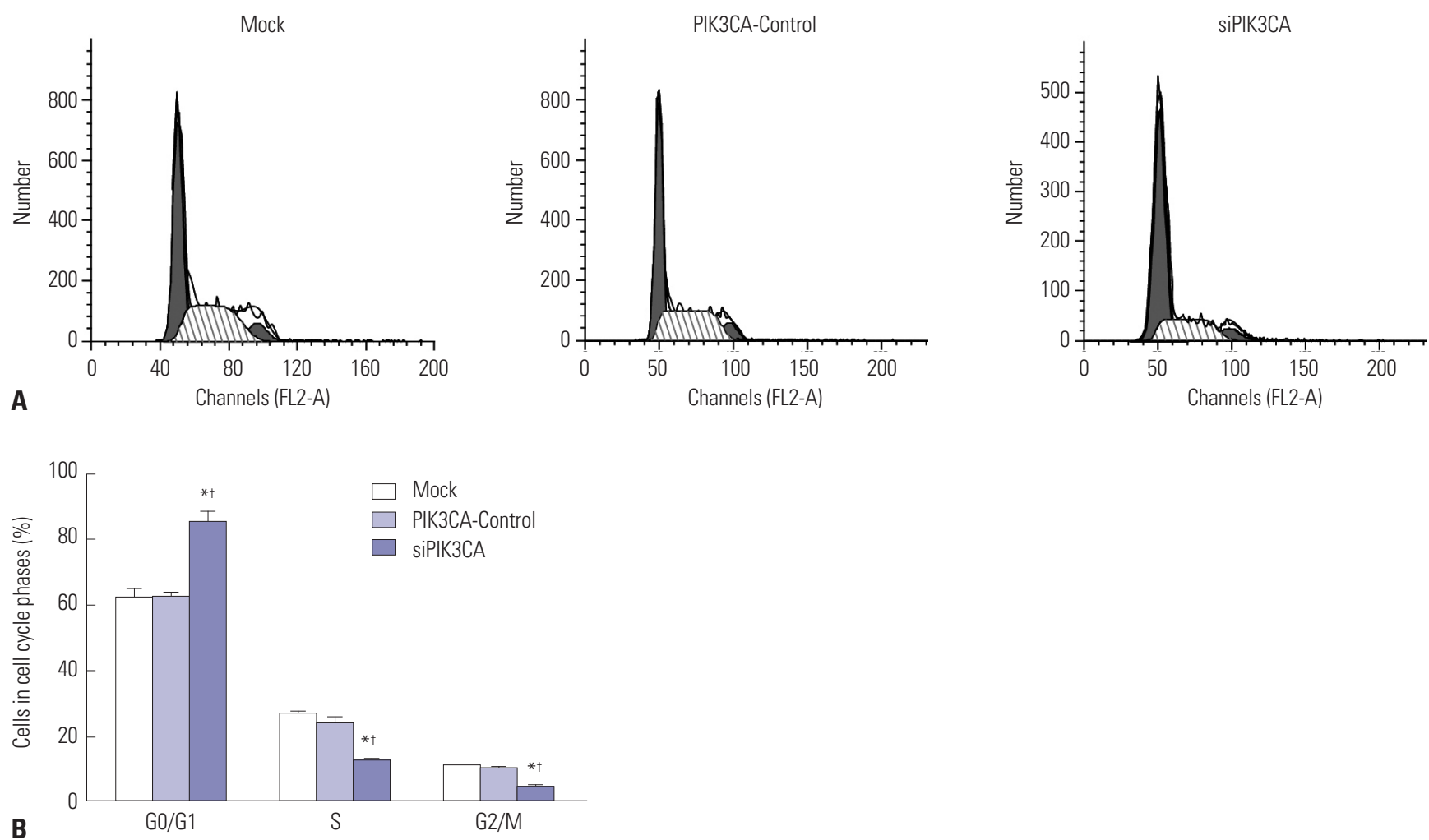

Fig. 4. PIK3CA silencing arrests Nalm-6 cells at G0/G1 phase. (A) Flow cytometry was used to detect cell cycle distributions of cells in each group. The process in this figure included G0/G1, S, and G2/M stage. (B) Statistics on the proportion of cells at different cell cycles of each group. * $p<0.05$ when compared with Mock group, ${ }^{\dagger} p<0.05$ when compared with PIK3CA-Control group. 
PIK3CA silencing inhibits Nalm-6 cells proliferation According to the results of MTT assay, from 1-4 days, lower OD495 values were observed in the siPIK3CA group than in the Mock group ( $p=0.023$ on the 1st day, $p=0.034$ on the 2 nd day, $p=0.027$ on the 3rd day, and $p=0.040$ on the 4 th day). At the same time, the OD495 value of the siPIK3CA group was still markedly lower than that of the PIK3CA-Control group ( $p=0.026$ on the 1st day, $p=0.039$ on the 2 nd day, $p=0.028$ on the 3rd day, and $p=0.044$ on the 4 th day). However, compared with the Mock group, the OD495 value of the PIK3CA-Control group was not obviously changed (Fig. 3). PIK3CA silencing inhibited Nalm- 6 cells proliferation.

\section{PIK3CA silencing arrests Nalm-6 cells in the G0/G1 phase}

Cell cycle distribution was detected by flow cytometry. Nalm6 cells of the Mock group and the PIK3CA-Control group had similar proportion of cells at G0/G1 phase, S phase, and G2/ $\mathrm{M}$ phase. However, a dramatically increased proportion of $\mathrm{G} 0 / \mathrm{Gl}$ phase $(p=0.020)$ cells and a remarkably decreased proportion of S phase $(p=0.037)$ and $\mathrm{G} 2 / \mathrm{M}$ phase $(p=0.023)$ cells was found in the siPIK3CA group when compared with the Mock group. In addition, when compared with the PIK3CAControl group, cells of the siPIK3CA group exhibited a much higher proportion of cells in the G0/G1 phase ( $p=0.022)$, as well as a remarkably lower proportion in the $S$ phase $(p=0.041)$ and $\mathrm{G} 2 / \mathrm{M}$ phase ( $p=0.026)$ (Fig. 4$)$. All of these data indicated that PIK3CA silencing could arrest Nalm-6 cells at the G0/G1 phase.

\section{PIK3CA silencing promotes Nalm-6 cells apoptosis}

Four days after transfection, apoptosis in each group was investigated by flow cytometry. Compared with the Mock group, obvious changes in percentages of apoptosis cells were not found in the PIK3CA-Control group; however, an aberrantly higher percentage of apoptosis cells was observed in the siPIK3CA group, compared to the Mock group $(p=0.016)$ and PIK3CA-Control group ( $p=0.022$ ) (Fig. 5). These results suggested that PIK3CA silencing could stimulate Nalm-6 cells apoptosis.

\section{PIK3CA silencing suppresses Nalm- $\mathbf{6}$ cells invasion}

The number of invasion cells in the PIK3CA-Control group was $135.0 \pm 5.77$, which was not markedly different from that in the Mock group (144.1 \pm 8.90$)$. Meanwhile, the number of invasion cells in the siPIK3CA group was only $48.9 \pm 9.27$, which was significantly lower than that in the Mock group ( $p=0.03$ ) and PIK3CA-Control group ( $p=0.025$ ) (Fig. 6 ), thereby demonstrating the inhibitory effect of PIK3CA silencing on Nalm-6 cells invasion ability.

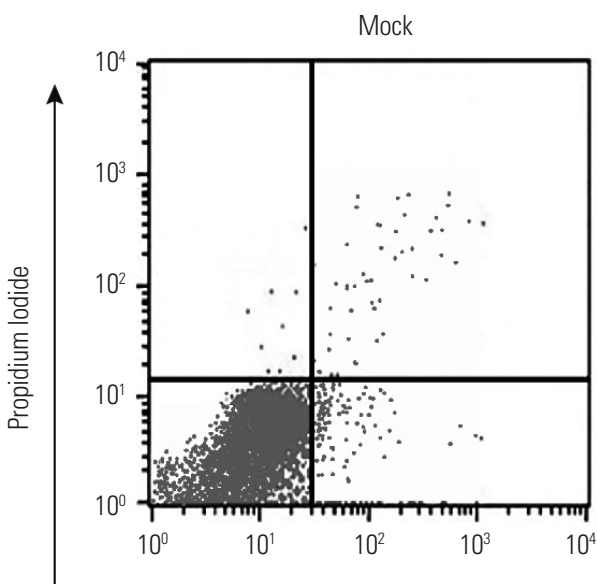

A
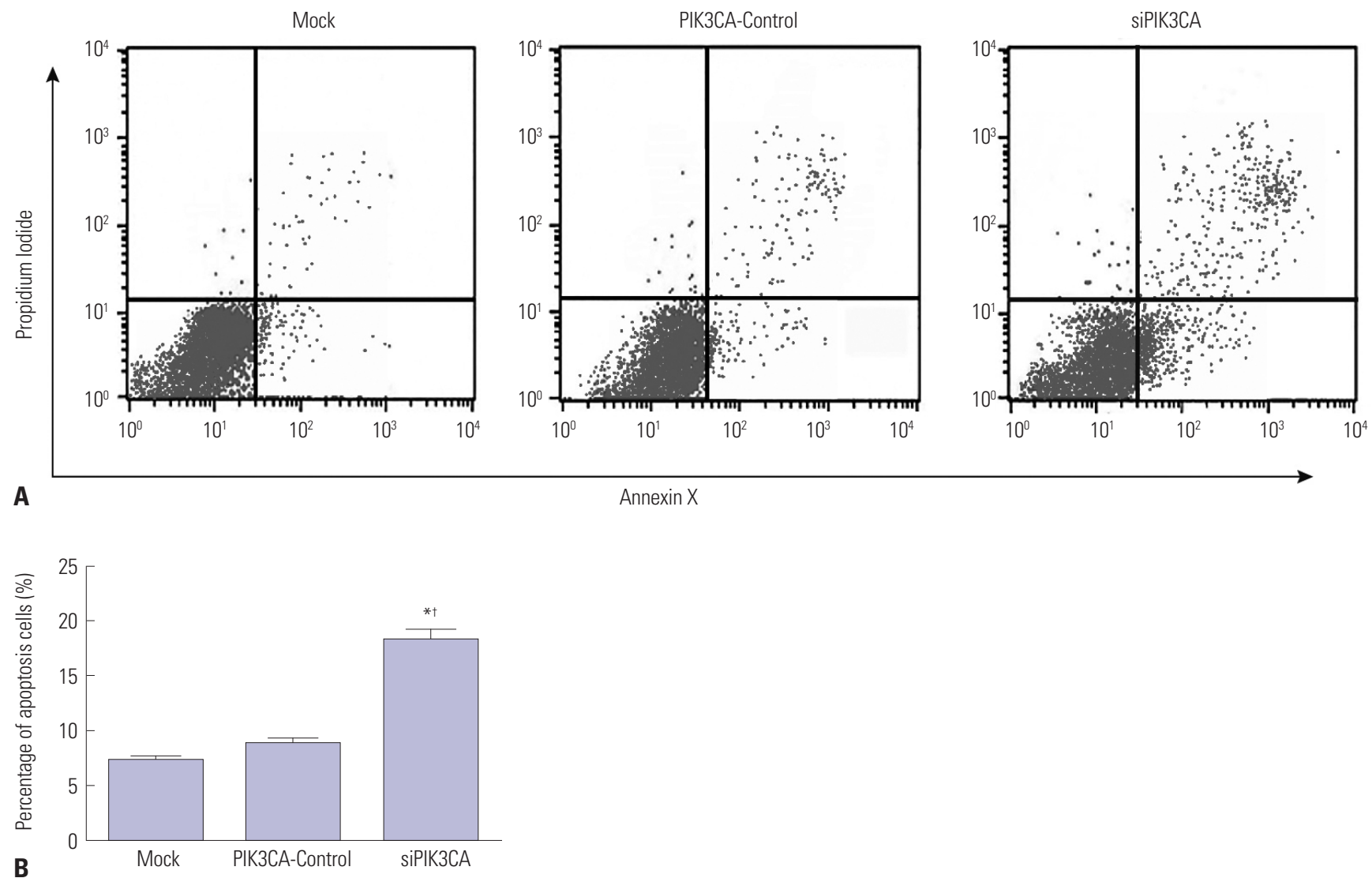

Fig. 5. PIK3CA silencing promotes Nalm-6 cell apoptosis. (A) Flow cytometry was used to detect apoptosis in each group. (B) Statistics on the percentage of apoptotic cells in each group. ${ }^{*} p<0.05$ when compared with Mock group, ${ }^{\dagger} p<0.05$ when compared with PIK3CA-Control group. 


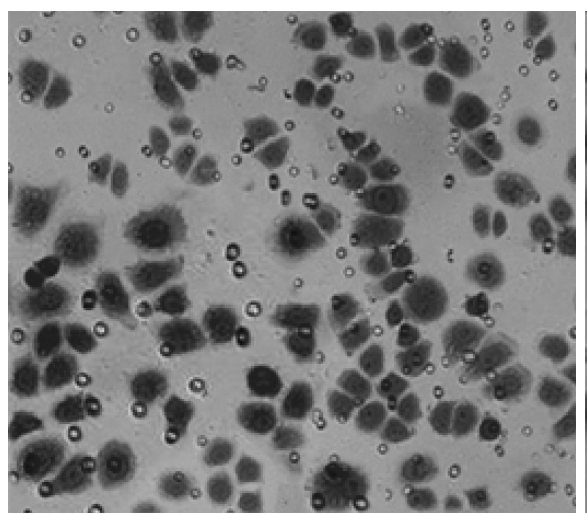

A

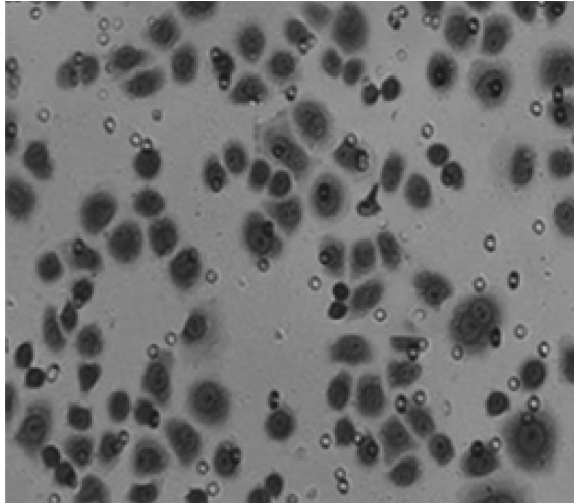

PIK3CA-Control

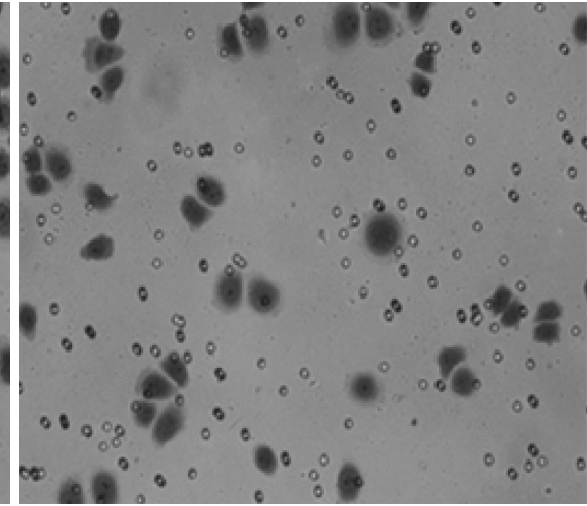

SiPIK3CA

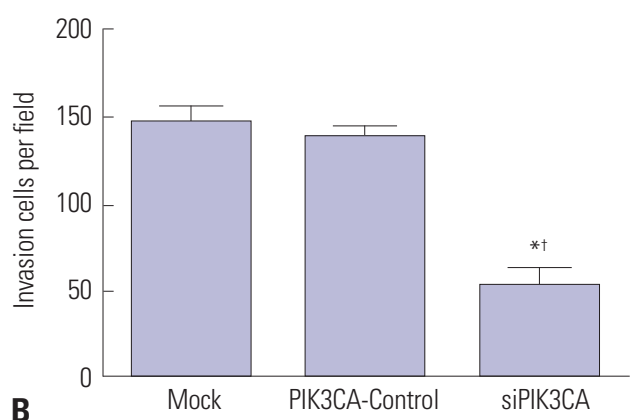

Fig. 6. PIK3CA silencing suppresses Nalm-6 cell invasion. (A) Crystal violet staining $(\times 300)$ of invasive cells in each group. (B) Statistics on the number of invasive cells in each group. ${ }^{*} p<0.05$ when compared with Mock group, ${ }^{\dagger} p<0.05$ when compared with PIK3CA-Control group.

\section{PIK3CA silencing enhances Nalm-6 cells sensitivity to chemotherapeutic drugs}

The inhibition rates of Nalm- 6 cells in the Mock group, PIK3CA-Control group, and siPIK3CA group all increased with time after treatment with VCR. In addition, the inhibition rate of cells in the siPIK3CA group was significantly higher than that in the Mock group ( $p=0.018$ on the 1st day, $p=0.020$ on the 2 nd day, $p=0.018$ on the 3rd day, and $p=0.037$ on the 4 th day) and the PIK3CA-Control group ( $p=0.019$ on the 1st day, $p=0.022$ on the 2 nd day, $p=0.019$ on the 3 rd day, and $p=0.042$ on the 4 th day) after being treated for 1-4 days. No obvious difference was found between the PIK3CA-Control group and Mock group at the same time (Fig. 7A). Furthermore, after being treated by DNR, there was no significant difference in inhibition rate of cells in the PIK3CA-Control group and Mock group. However, cells in the siPIK3CA group exhibited much higher inhibition at 1-4 days, compared with the Mock group ( $p=0.029$ on the 1st day, $p=0.024$ on the 2 nd day, $p=0.031$ on the 3rd day, and $p=0.033$ on the 4 th day) and PIK3CA-Control group ( $p=0.031$ on the 1st day, $p=0.027$ on the 2nd day, $p=0.037$ on the 3rd day, and $p=0.032$ on the 4 th day) (Fig. 7B). Therefrom, it could be concluded that PIK3CA silencing enhances Nalm- 6 cell sensitivity to chemotherapeutic drugs.

\section{PIK3CA silencing inhibits the phosphorylation of Akt} Western blot results showed that the relative Akt protein expression in the PIK3CA-Control group, siPIK3CA group, and
siPIK3CA+Akti-1/2 group was similar to that in Mock group. pAkt relative expression in the PIK3CA-Control group was also not significantly changed when compared with the Mock group. However, much decreased pAkt relative expression was observed in the siPIK3CA group ( $p=0.026$ and $p=0.031$ ) and siPIK3CA+Akti- $1 / 2$ group ( $p=0.019$ and $p=0.023$ ) when compared with the Mock group and PIK3CA-Control group (Fig. 8). The results above revealed that PIK3CA silencing inhibited the phosphorylation of Akt, which could suppress the activity of PI3K/Akt signaling.

\section{DISCUSSION}

Leukemia, especially acute B lymphocytic leukemia, is the most common malignant tumor in children. ${ }^{16}$ Chemotherapy still remains the main method for the treatment of leukemia currently, although a large amount of patients show poor responses to chemotherapy. ${ }^{17-19}$ The development of drug resistance among leukemia cells is the main cause of chemotherapy failure in leukemia patients, which adversely affects prognosis in patients with leukemia. ${ }^{20}$ Studies have shown that chemoresistance is associated with decreased sensitivity of tumor cells to apoptosis. ${ }^{21,22}$ In this study, we discovered that PIK3CA expression is increased in Nalm- 6 cells and that PIK3CA silencing enhances the sensitivity of Nalm-6 cells to chemotherapy drugs (VCR and DNR) by suppressing the phosphorylation of Akt. 

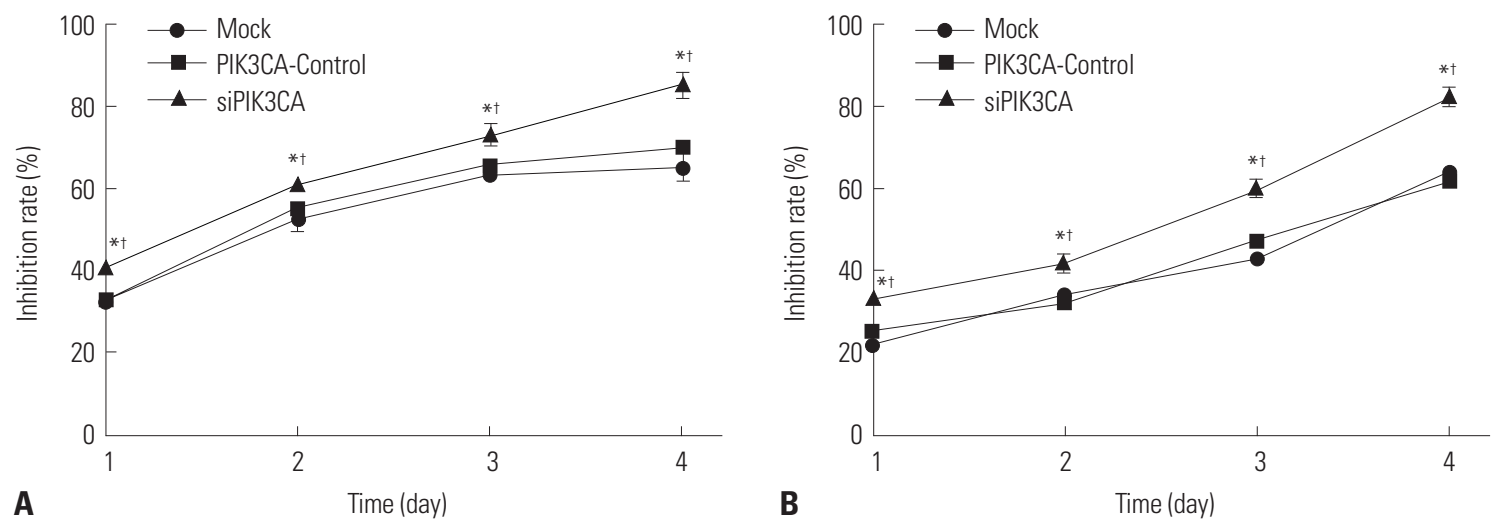

Fig. 7. PIK3CA silencing enhances Nalm-6 cell sensitivity to chemotherapeutic drugs. (A) Inhibition rate of cells in each group after being treated by vincristine. (B) Inhibition rate of cells in each group after daunorubicin treatment. ${ }^{*} p<0.05$ when compared with Mock group at the same time, ${ }^{\dagger} p<0.05$ when compared with PIK3CA-Control group.
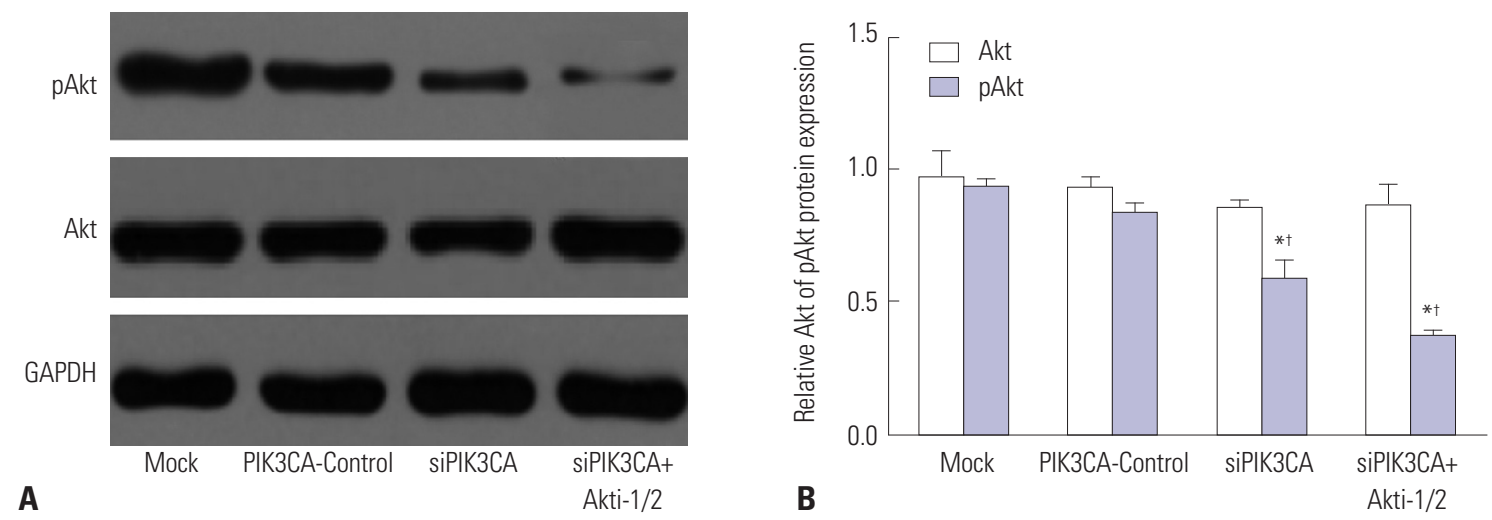

Fig. 8. PIK3CA silencing inhibits the phosphorylation of Akt. (A) Western blot of proteins in each group. (B) Statistical graph of proteins relative expression in each group. ${ }^{*} p<0.05$ when compared with Mock group, ${ }^{\dagger} p<0.05$ when compared with PIK3CA-Control group.

PIK3CA, defined as an oncogene, is the catalytic subunit of PI3K, the mutation or amplification of which could promote cells proliferation and inhibit their apoptosis by regulating the PI3K/Akt pathway. ${ }^{23,24}$ PIK3CA was found to be up-regulated in a variety of tumors and to promote the progression of multiple tumors. For instance, Zardavas, et al. ${ }^{25}$ investigated the association of PIK3CA mutation with prognosis in breast cancer patients by a summary analysis of individual patient data. They noticed that, of all 10319 breast cancer patients, up to 32\% exhibited PIK3CA mutation, and a significant correlation was found between PIK3CA mutation and ER positivity or lower grade. Little is known about PIK3CA mutations in hematological malignancies. Cui, et al. ${ }^{26}$ reported that PIK3CA was extensively expressed in diffuse large B cell lymphoma. They also observed that, after PIK3CA being knockdown, diffuse large B cell lymphoma cells proliferation ability was markedly impaired and that apoptosis was obviously enhanced. At the same time, pronouncedly declined p-AKT expression also occurred by silencing PIK3CA. Abubaker, et al. ${ }^{27}$ also indicated that, among 215 cases of diffuse large B cell lymphoma patients, 17 cases (18\%) exhibited PIK3CA mutation. Our results also revealed remarkable increases in PIK3CA expression in Nalm-6 cells, which is consistent with previous studies.
Furthermore, the results from this research also revealed that, after being transfected by PIK3CA siRNA, the proliferation and invasion ability of Nalm- 6 cells were suppressed, while their apoptosis ability was enhanced. At the same time, significantly enhanced sensitivity to chemotherapeutic drugs (VCR and DNR) was found after PIK3CA was silenced in Nalm-6 cells. Drug resistance among tumor cells was thought to be caused mainly by a combination of multiple anti-apoptotic genes, and improving the sensitivity of tumor cells to drugs was one of the important strategies to improve the efficacy of chemotherapy. ${ }^{28}$ Black and colleagues ${ }^{29}$ had shown that PIK3CA mutation could cause resistance to drugs, such as trastuzumab, among uterine serous carcinomas tumor cells. Patra, et al. ${ }^{30}$ also evaluated the sensitivity of breast cancer cells to trastuzumab and found an reduced sensitivity to trastuzumab for breast cancer cells. Ross, et al. ${ }^{31}$ pointed out in their study that, once PIK3CA in urothelial carcinomas cells was knockdown by retroviral-mediated shRNA delivery, the proliferation and migration ability of these urothelial carcinomas cells was significantly impaired. Our research further validated the results of these precursor studies, demonstrating that PIK3CA silencing is helpful to increase Nalm-6 cell sensitivity to chemotherapeutic drugs (VCR and DNR). Under normal 
physiological conditions, PIK3CA is expressed in normal brain, lung, breast, gastrointestinal, cervix, ovary and other tissues. It has many important physiological functions, such as regulating somatic cell proliferation, differentiation, and survival. However, under normal circumstances, PIK3CA exists in an inactive form and is hardly detected. ${ }^{32}$ After mutation, PIK3CA and its protein are easily detected due to being overexpressed, which are considered to contribute to cell carcinogenesis. ${ }^{33}$

In the present study, we noticed that PIK3CA silencing might promote Nalm-6 cell sensitivity to chemotherapeutic drugs by suppressing the phosphorylation of Akt. Akt, one of the important genes in the PI3K/Akt pathway, which plays a vital role in the progression of a variety of tumors. ${ }^{34,35}$ In recent years, research on the effects of Akt on tumor cell drug sensitivity has accumulated. These studies have shown that inhibition of Akt phosphorylation could enhance the sensitivity of gastric cancer cell lines to cisplatin, thereby increasing the therapeutic effect of cisplatin on gastric cancer. ${ }^{36}$ Mutation of PIK3CA results in overexpression of PIK3CA protein, which leads to an increase in the catalytic activity of PI3K. Then, activated PI3K/AKT signalling further promotes cell carcinogenesis. ${ }^{37,38}$ Samuels, et al. ${ }^{39}$ constructed a cancer cell line with PIK3CA gene mutation in vitro. Their study revealed that PI3K kinase activity is significantly enhanced after PIK3CA mutation and that the phosphorylation level of AKT was also dramatically increased. AKT is a downstream direct target protein of PI3K, and its phosphorylation is closely related to the activation state of PI3K. Phosphorylated AKT could promote phosphorylation of multiple substrates downstream, thereby promoting tumor growth via inhibiting apoptosis and stimulating proliferation. ${ }^{40}$ This study confirmed for the first time that silencing of PIK3CA enhances chemotherapeutic drugs sensitivity Nalm-6 cells by inhibiting the phosphorylation of Akt. Clinically, it might be possible to inhibit the occurrence of leukemia and enhance sensitivity to chemotherapeutic drugs by silencing PIK3CA expression.

In conclusion, this study indicated that PIK3CA silencing could inhibit Nalm-6 cell proliferation and invasion and enhance Nalm-6 cell apoptosis and sensitivity to chemotherapeutic drugs (VCR and DNR) by suppressing the phosphorylation of Akt. This article provides potential therapeutic targets for the treatment of childhood leukemia.

\section{AUTHOR CONTRIBUTIONS}

Conceptualization: Xiuling Liang. Data curation: Xiuling Liang, Xianfang Xin. Formal analysis: Xiuling Liang. Investigation: Xianfang Xin. Project administration: Xiuling Liang, Chengyan Fu, Mingde Ding. Writing_original draft: Xiuling Liang, Xianfang Xin, Dongmei Qi, Chengyan Fu, Mingde Ding. Writing - review \& editing: Xiuling Liang, Xianfang Xin, Dongmei Qi, Chengyan Fu, Mingde Ding.

\section{ORCID iDs}

Xiuling Liang

Xianfang Xin

Dongmei Qi

Chengyan Fu

Mingde Ding

https://orcid.org/0000-0002-2868-9642

https://orcid.org/0000-0002-6796-9436

https://orcid.org/0000-0001-9350-6235

https://orcid.org/0000-0002-1181-5286

https://orcid.org/0000-0002-5629-0858

\section{REFERENCES}

1. Farah RA, Horkos JG, Bustros YD, Farhat HZ, Abla O. A multicenter experience from Lebanon in childhood and adolescent acute myeloid leukemia: high rate of early death in childhood acute promyelocytic leukemia. Mediterr J Hematol Infect Dis 2015;7:e2015012.

2. Yang R, Zhong L, Yang XQ, Jiang KL, Li L, Song H, et al. Neutrophil elastase enhances the proliferation and decreases apoptosis of leukemia cells via activation of PI3K/Akt signaling. Mol Med Rep 2016; 13:4175-82.

3. Singh G, Parmar P, Kataria S, Singh S, Sen R. Spectrum of acute and chronic leukemia at a tertiary care hospital, Haryana, India. Int J Res Med Sci 2016;4:1115-8.

4. Barrington-Trimis JL, Cockburn M, Metayer C, Gauderman WJ, Wiemels J, McKean-Cowdin R. Trends in childhood leukemia incidence over two decades from 1992 to 2013. Int J Cancer 2017;140: 1000-8.

5. Janitz AE, Campbell JE, Magzamen S, Pate A, Stoner JA, Peck JD. Traffic-related air pollution and childhood acute leukemia in Oklahoma. Environ Res 2016;148:102-11.

6. Carew JS, Zhou Y, Albitar M, Carew JD, Keating MJ, Huang P. Mitochondrial DNA mutations in primary leukemia cells after chemotherapy: clinical significance and therapeutic implications. Leukemia 2003;17:1437-47.

7. Hsiao YL, Chang PC, Huang HJ, Kuo CC, Chen CY. Treatment of acute lymphoblastic leukemia from traditional chinese medicine. Evid Based Complement Alternat Med 2014;2014:601064.

8. Rodríguez-Lirio A, Pérez-Yarza G, Fernández-Suárez MR, AlonsoTejerina E, Boyano MD, Asumendi A. Metformin induces cell cycle arrest and apoptosis in drug-resistant leukemia cells. Leuk Res Treatment 2015;2015:516460.

9. Macanas-Pirard P, Broekhuizen R, González A, Oyanadel C, Ernst D, García P, et al. Resistance of leukemia cells to cytarabine chemotherapy is mediated by bone marrow stroma, involves cellsurface equilibrative nucleoside transporter-1 removal and correlates with patient outcome. Oncotarget 2017;8:23073-86.

10. Wang L, Chen B, Lin M, Cao Y, Chen Y, Chen X, et al. Decreased expression of nucleophosmin/B23 increases drug sensitivity of adriamycin-resistant Molt-4 leukemia cells through mdr-1 regulation and Akt/mTOR signaling. Immunobiology 2015;220:331-40.

11. Xu H, Li Y, Chen L, Wang C, Wang Q, Zhang H, et al. SIRT2 mediates multidrug resistance in acute myelogenous leukemia cells via ERK1/2 signaling pathway. Int J Oncol 2016;48:613-23.

12. Jiang Q, Lu X, Huang P, Gao C, Zhao X, Xing T, et al. Expression of miR-652-3p and effect on apoptosis and drug sensitivity in pediatric acute lymphoblastic leukemia. Biomed Res Int 2018;2018: 5724686.

13. Liang H, Zheng QL, Fang P, Zhang J, Zhang T, Liu W, et al. Targeting the PI3K/AKT pathway via GLI1 inhibition enhanced the drug sensitivity of acute myeloid leukemia cells. Sci Rep 2017;7:40361.

14. Gu M, Nishihara R, Chen Y, Li W, Shi Y, Masugi Y, et al. Aspirin exerts high anti-cancer activity in PIK3CA-mutant colon cancer cells. Oncotarget 2017;8:87379-89.

15. Zheng S, Yang C, Lu M, Liu Q, Liu T, Dai F, et al. PIK3CA promotes 
proliferation and motility but is unassociated with lymph node metastasis or prognosis in esophageal squamous cell carcinoma. Hum Pathol 2016;53:121-9.

16. Zhang Y, Gao Y, Shi R, Chen D, Wang X, Kamijima M, et al. Household pesticide exposure and the risk of childhood acute leukemia in Shanghai, China. Environ Sci Pollut Res Int 2015;22:11755-63.

17. Boles JM, Dutel JL, Briere J, Mialon P, Robasckiewicz M, Garre M, et al. Acute renal failure caused by extreme hyperphosphatemia after chemotherapy of an acute lymphoblastic leukemia. Cancer 1984;53:2425-9.

18. Appelbaum FR, Kopecky KJ. Long-term survival after chemotherapy for acute myeloid leukemia: the experience of the Southwest Oncology Group. Cancer 1997;80(11 Suppl):2199-204.

19. Xu P, Wang M, Jiang Y, Ouyang J, Chen B. The association between expression of hypoxia inducible factor- $1 \alpha$ and multi-drug resistance of acute myeloid leukemia. Transl Cancer Res 2017;6:198-205.

20. Gupta R, Chandgothia M, Dahiya M, Bakhshi S, Sharma A, Kumar L. Multi-drug resistance protein 1 as prognostic biomarker in clinical practice for acute myeloid leukemia. Int J Lab Hematol 2016; 38:e93-7.

21. Rubio MF, Lira MC, Rosa FD, Sambresqui AD, Salazar Güemes MC, Costas MA. RAC3 influences the chemoresistance of colon cancer cells through autophagy and apoptosis inhibition. Cancer Cell Int 2017;17:111.

22. Bhola PD, Mar BG, Lindsley RC, Ryan JA, Hogdal LJ, Vo TT, et al. Functionally identifiable apoptosis-insensitive subpopulations determine chemoresistance in acute myeloid leukemia. J Clin Invest 2016;126:3827-36.

23. Peng Y, Zhang X, Ma Q, Yan R, Qin Y, Zhao Y, et al. MiRNA-194 activates the Wnt/ $\beta$-catenin signaling pathway in gastric cancer by targeting the negative Wnt regulator, SUFU. Cancer Lett 2017;385: 117-27.

24. Henry WS, Laszewski T, Tsang T, Beca F, Beck AH, McAllister SS, et al. Aspirin suppresses growth in PI3K-mutant breast cancer by activating AMPK and inhibiting mTORC1 signaling. Cancer Res 2017;77:790-801.

25. Zardavas D, Te Marvelde L, Milne RL, Fumagalli D, Fountzilas G, Kotoula V, et al. Tumor PIK3CA genotype and prognosis in earlystage breast cancer: a pooled analysis of individual patient data. J Clin Oncol 2018;36:981-90.

26. Cui W, Zheng S, Liu Z, Wang W, Cai Y, Bi R, et al. PIK3CA expression in diffuse large B cell lymphoma tissue and the effect of its knockdown in vitro. Onco Targets Ther 2017;10:2239-47.

27. Abubaker J, Bavi PP, Al-Harbi S, Siraj AK, Al-Dayel F, Uddin S, et al. PIK3CA mutations are mutually exclusive with PTEN loss in diffuse large B-cell lymphoma. Leukemia 2007;21:2368-70.
28. Burger H, Foekens JA, Look MP, Meijer-van Gelder ME, Klijn JG, Wiemer EA, et al. RNA expression of breast cancer resistance protein, lung resistance-related protein, multidrug resistance-associated proteins 1 and 2, and multidrug resistance gene 1 in breast cancer: correlation with chemotherapeutic response. Clin Cancer Res 2003;9:827-36.

29. Black JD, Lopez S, Cocco E, Bellone S, Altwerger G, Schwab CL, et al. PIK3CA oncogenic mutations represent a major mechanism of resistance to trastuzumab in HER2/neu overexpressing uterine serous carcinomas. Br J Cancer 2015;113:1020-6.

30. Patra S, Young V, Llewellyn L, Senapati JN, Mathew J. BRAF, KRAS and PIK3CA mutation and sensitivity to trastuzumab in breast cancer cell line model. Asian Pac J Cancer Prev 2017;18:2209-13.

31. Ross RL, McPherson HR, Kettlewell L, Shnyder SD, Hurst CD, Alder O, et al. PIK3CA dependence and sensitivity to therapeutic targeting in urothelial carcinoma. BMC Cancer 2016;16:553.

32. Broderick DK, Di C, Parrett TJ, Samuels YR, Cummins JM, McLendon RE, et al. Mutations of PIK3CA in anaplastic oligodendrogliomas, high-grade astrocytomas, and medulloblastomas. Cancer Res 2004;64:5048-50.

33. Rameh LE, Cantley LC. The role of phosphoinositide 3-kinase lipid products in cell function. J Biol Chem 1999;274:8347-50.

34. Jiang C, Xu R, Li XX, Wang YY, Liang WQ, Zeng JD, et al. p53R2 overexpression in cervical cancer promotes AKT signaling and EMT, and is correlated with tumor progression, metastasis and poor prognosis. Cell Cycle 2017;16:1673-82.

35. Tian B, Chen X, Zhang H, Li X, Wang J, Han W, et al. Urokinase plasminogen activator secreted by cancer-associated fibroblasts induces tumor progression via PI3K/AKT and ERK signaling in esophageal squamous cell carcinoma. Oncotarget 2017;8:4230013.

36. Zhou L, Wu Y, Guo Y, Li Y, Li N, Yang Y, et al. Calycosin enhances some chemotherapeutic drugs inhibition of Akt signaling pathway in gastric cells. Cancer Invest 2017;35:289-300.

37. Fang WL, Huang KH, Lan YT, Lin CH, Chang SC, Chen MH, et al. Mutations in PI3K/AKT pathway genes and amplifications of PIK3CA are associated with patterns of recurrence in gastric cancers. Oncotarget 2016;7:6201-20.

38. Ito C, Nishizuka SS, Ishida K, Uesugi N, Sugai T, Tamura G, et al. Analysis of PIK3CA mutations and PI3K pathway proteins in advanced gastric cancer. J Surg Res 2017;212:195-204.

39. Samuels Y, Wang Z, Bardelli A, Silliman N, Ptak J, Szabo S, et al. High frequency of mutations of the PIK3CA gene in human cancers. Science 2004;304:554.

40. Li Y, Li J. Relationship between PI3K/AKT/PTEN pathway and lung cancer. J Qiqihar Med College 2013;34:2657-9. 